\title{
L'appareil musculaire régit le développement squelettique
}

\author{
Eckhard Schoenau \\ Hôpital d'enfants, Université de Cologne, Cologne, Allemagne
}

\author{
Mots-clés \\ Résistance osseuse - Force musculaire - Ostéoporose, \\ prévention · Sarcopénie
}

\section{Résumé}

La présente analyse traite de concepts méthodologiques dans l'évaluation de la fonction des muscles squelettiques et de l'adaptation osseuse. La propriété capitale de l'os est aujourd'hui considérée comme étant la résistance et non le poids, et le contrôle de cette résistance paraît être principalement dû à l'effet des charges mécaniques s'exerçant sur l'os. La contraction musculaire place la charge physiologique la plus élevée sur l'os et, de ce fait, la stabilité osseuse doit être adaptée à la force musculaire (unité fonctionnelle ostéomusculaire). Les suggestions et recommandations décrites soulignent un nouveau concept: la masse et la résistance osseuses ne doivent pas être reliées à l'âge mais, comme le montrent des données de plus en plus nombreuses, elles pourraient désormais être liées à la fonction musculaire. Selon cette analyse, il n'existe aucune entité correspondant à une «masse osseuse maximale». De nombreuses études sont en cours afin d'évaluer si ces nouvelles approches accroissent la sensibilité et la spécificité du pronostic de fracture chez un sujet. De plus, le domaine de recherche de nombreux investigateurs en matière de physiologie osseuse se déplace de la masse osseuse vers la géométrie ou la résistance des os et vers leur relation à l'appareil musculaire qui les régit.

Copyright @ 2006 Nestec Ltd., Vevey/S. Karger AG, Basel (c) 2006 Nestec Ltd., Vevey/S. Karger AG, Basel 0250-9644/06/0642-0055\$23.50/0

Fax +41 613061234

E-Mail karger@karger.ch

www.karger.com
Accessible en ligne à: www.karger.com/anf

\section{Introduction}

Chaque génération pose de nouveaux problèmes au système de santé par de nouvelles demandes, tandis que la prévalence des troubles médicaux nécessitant des approches préventives et curatives se modifie constamment. Dans les pays occidentaux industrialisés, la plupart des sujets ont remplacé les activités motrices de la vie quotidienne, précédemment fréquentes, par l'assistance technique offerte par un monde mécanisé et informatisé. L'appareil locomoteur s'adaptant aux épreuves biomécaniques et aux conditions environnementales, la composition corporelle moyenne de l'enfant et de l'adolescent a très fortement changé dans la société occidentale $[1,2]$. Une insulinorésistance, une obésité, une ostéopénie et une sarcopénie sont aujourd'hui les problèmes typiques auxquels sont confrontés les professionnels de santé œuvrant dans le domaine de la prévention et de l'éducation pour la santé. Jusqu'il y a quelques décennies, dans les sociétés occidentales, les soins pédiatriques étaient centrés sur la prévention du rachitisme et de la malnutrition dus à des apports caloriques insuffisants. De nos jours, les problèmes urgents sont une atrophie de l'appareil locomoteur due à une faible activité motrice et à une malnutrition liée à des apports caloriques élevés. De plus, l'amélioration des soins des enfants atteints d'une maladie chronique a introduit le problème des affections musculosquelettiques secondaires. De ce fait, les connaissances relatives à l'évaluation des interactions entre muscles et squelette et le savoir-faire en ce domaine deviendront 
Fig. 1. Modèle fonctionnel de développement osseux basé sur la théorie du mécanostat. La pièce centrale de la régulation osseuse est la boucle de rétroaction entre la déformation (contrainte tissulaire) et la résistance de l'os. Au cours de la croissance, ce système homéostatique doit constamment s'adapter à des contraintes externes. Les facteurs présentés au-dessous modulent divers aspects du système régulateur central. D'après Rauch et Schoenau [29], avec autorisation.

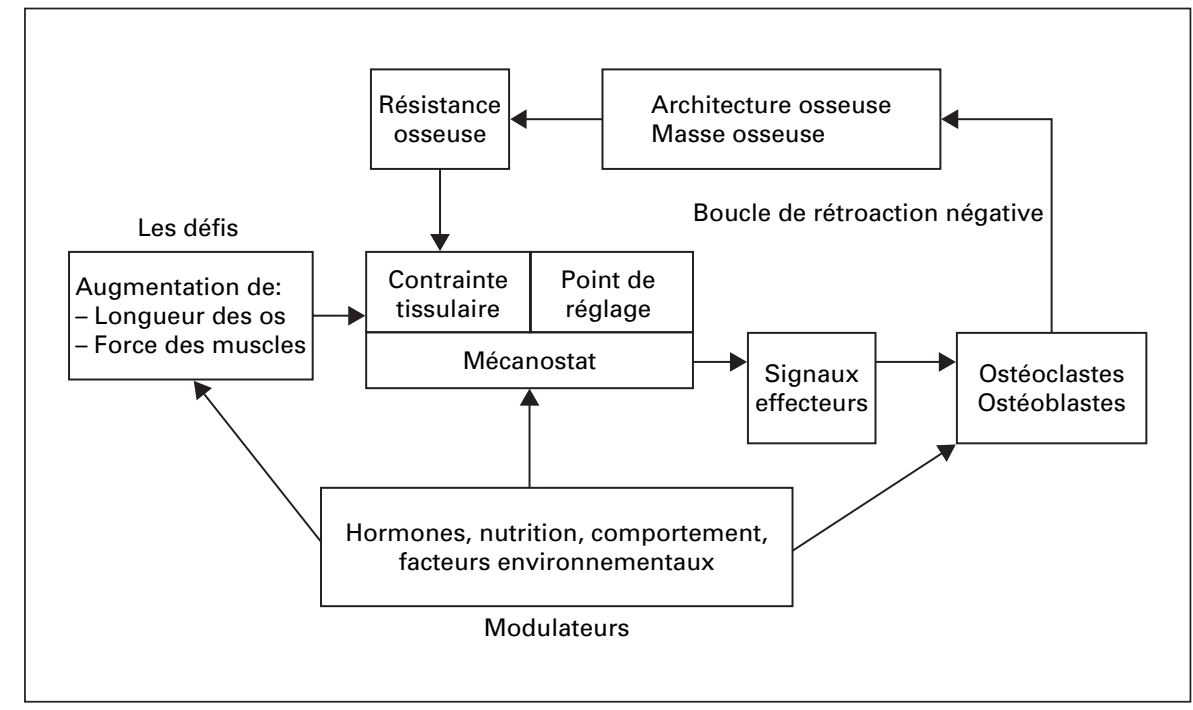

de plus en plus un centre d'intérêt en pédiatrie. Les connaissances relatives au développement des systèmes musculosquelettiques et l'évaluation de ce processus deviendront des domaines importants pour les personnes responsables de l'amélioration de la qualité des soins médicaux dans nos sociétés. La présente analyse de la littérature discute des propriétés mécaniques du développement squelettique.

\section{L'hypothèse du mécanostat de Harold Frost}

L'association des facteurs qui assurent la santé des os porteurs satisfait aux besoins de tous les amphibiens, oiseaux, mammifères et reptiles quels que soient leur taille, âge et sexe, et a été appelée mécanostat. Ce dernier associe des mécanismes de modelage et de remodelage, leurs seuils, les mécanismes médiateurs médullaires, les mécanismes de signalement qui les connectent et peutêtre d'autres facteurs. Pour des raisons mécaniques, le système de rétroaction négative qui en résulte détermine si, quand et où les os nécessitent plus de résistance, ou quand du tissu osseux n'est pas nécessaire. Divers facteurs non mécaniques, dont des hormones et d'autres agents humoraux, pourraient moduler («accroître ou réduire») les effets du mécanostat sur la résistance osseuse. Le mécanostat pourrait être comparé à l'association du volant, des freins et de l'accélérateur d'une automobile. Les ostéoblastes et les ostéoclastes pourraient être comparés à ses roues, et l'utilisation mécanique à son conducteur (fig. 1) [3-5].
Le développement des muscles et des os au cours de la croissance est influencé par des forces associées à la gravité et à l'activité physique $[4,6,7]$. Ce sont les forces musculaires qui créent les forces maximales agissant sur l'os. De ce fait, la croissance en l'absence de charge résulte à la fois en des muscles dépourvus de capacité fonctionnelle et en des os ne présentant pas la forme spécifique indispensable à leur fonction [8]. Cette relation intrinsèque entre le muscle et l'os est décrite par la théorie du mécanostat, qui suppose qu'une augmentation de la force musculaire maximale au cours de la croissance ou en réponse à une mise en charge croissante influence la masse, les dimensions et la résistance osseuses. La mise en décharge (non-utilisation ou immobilisation) aboutit à une réduction du développement et de la force musculaires, qui exerce un effet négatif sur ces trois derniers paramètres.

Le fonctionnement correct du mécanostat dépend de la normalité de la totalité de ses cellules (ostéocytes, ostéoblastes et ostéoclastes), de l'utilisation mécanique habituelle du squelette et de l'environnement endocrinien et métabolique [9]. L'ajustement fin du mécanostat est obtenu par des points physiologiques de réglage qui agissent à titre de seuil pour l'instauration ou l'inhibition du modelage et du remodelage osseux. Les points de réglage du mécanostat sont génétiquement déterminés, mais sont régulés par l'environnement endocrinien. Par exemple, il a été proposé qu'une réduction de la concentration des œstrogènes pouvait augmenter les points de réglage concernant le modelage et le remodelage osseux. L'environnement endocrinien affecte la sensibilité du mécanostat, avec laquelle l'os adapte sa masse, sa géométrie ou ses 


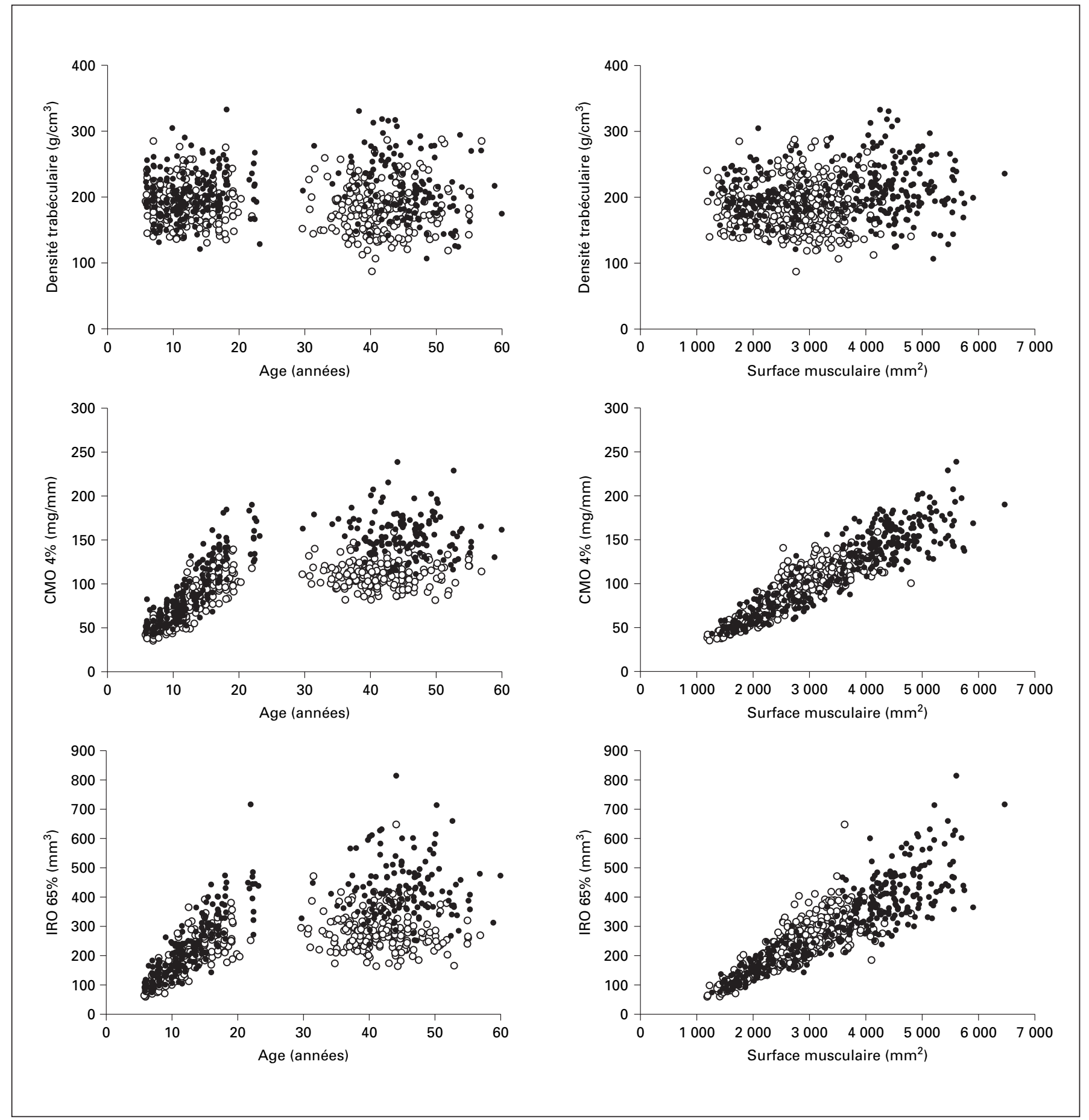

Fig. 2. Densité trabéculaire, contenu minéral osseux (CMO 4\%) et indice de résistance osseuse (IRO 65\%) par rapport à l'âge et à la surface musculaire. $\bigcirc=$ Femmes; $O$ = hommes. D'après Schoenau [13], avec autorisation. 


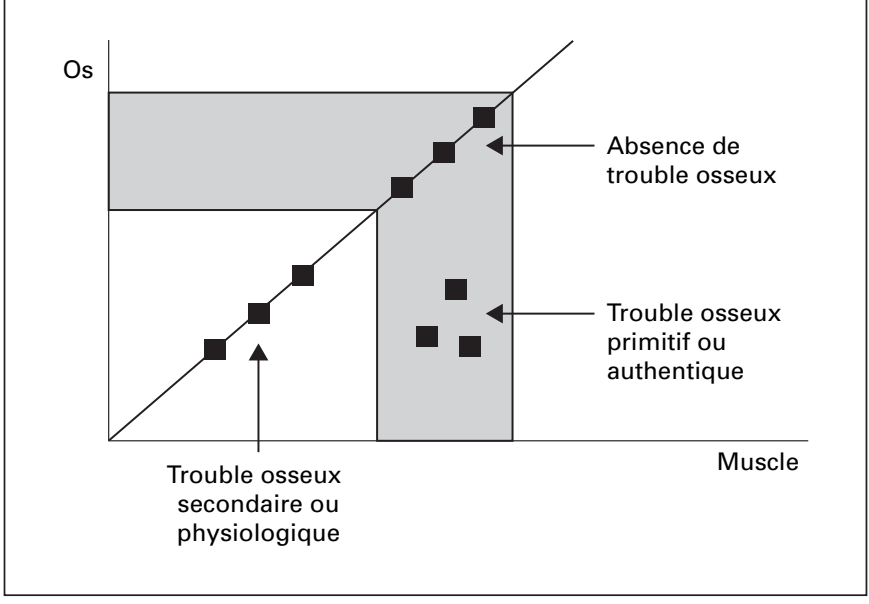

Fig. 3. «Unité fonctionnelle ostéomusculaire». Trouble osseux primitif: structure/masse osseuse non adaptée au développement musculaire. Trouble osseux secondaire: perturbation du développement musculaire mais squelette normal adapté. D'après Schoenau [13], avec autorisation.

propriétés structurales aux déformations induites par une mise en charge [9].

L'activité physique et la nutrition sont des facteurs environnementaux capitaux connus comme influençant le développement musculaire et osseux. L'exercice physique agit directement par action musculaire et indirectement par régulation endocrinienne; au cours de la croissance, l'exercice paraît influencer tant le modelage que la géométrie des os.

\section{Développement de la densité, de la masse et de la résistance osseuses}

La figure 2 montre la relation entre l'âge et la surface musculaire d'une part et la densité trabéculaire, la masse osseuse (contenu minéral osseux, CMO) et l'indice de résistance osseuse (IRO) d'autre part dans une population de référence en bonne santé. Les données anthropométriques et les résultats obtenus par tomodensitométrie quantitative périphérique chez ces sujets ont été précédemment publiés [10-12]. La densité trabéculaire à titre d'indice de la densité tissulaire ne dépend pas de l'âge ou du développement musculaire. Le CMO et l'IRO semblent dépendre de l'âge uniquement au cours de l'enfance. En revanche, ces deux paramètres sont en étroite corrélation linéaire avec le développement musculaire au cours de l'enfance et de l'âge adulte. Ces données indiquent que la densité osseuse est plus ou moins une "constante» et que le CMO et l'IRO sont des fonctions du développement musculaire. Sur la base de ces considérations, nous avons recommandé voici quelques années de relier les données osseuses analysées à des marqueurs indirects du développement musculaire. Par rapport aux données de référence liées à l'âge, l'analyse de «l'unité ostéomusculaire» (fig. 3) devrait améliorer les connaissances de la physiologie et de la physiopathologie du développement osseux [13].

\section{Influence de la puberté sur «l'unité ostéomusculaire»}

En 1995, Frost (communication personnelle) a soulevé une importante question: les œstrogènes pourraient-ils susciter une formation osseuse plus élevée que nécessaire aux activités physiques chez la femme, afin de stocker du calcium supplémentaire en prévision d'une gestation et d'un allaitement ultérieurs? Les données de Zanchetta et coll. [14] sont en faveur de cette hypothèse. Cette équipe a utilisé l'absorptiométrie biphotonique par rayons $\mathrm{X}$ afin d'estimer le CMO total et la masse maigre corporelle chez 778 enfants caucasiens en bonne santé vivant en Argentine. Ces données ont été réanalysées par Schiessl et coll. [15], qui ont montré qu'à la puberté, la masse osseuse des jeunes filles commençait à augmenter davantage que celle des garçons présentant une masse maigre similaire. En raison d'incertitudes méthodologiques et analytiques concernant l'utilisation du CMO total et de la masse maigre à titre d'indices de la résistance osseuse et de la force musculaire, et également de l'importance potentielle de ces observations, des études supplémentaires ont semblé nécessaires afin de les confirmer. Lors de l'étude nutritionnelle et anthropométrique longitudinale de Dortmund, la surface corticale du radius à titre d'indice de résistance osseuse et la surface musculaire représentant la force des muscles ont été mesurées par tomodensitométrie quantitative périphérique chez des enfants et des adolescents en bonne santé âgés de 6-22 ans et des adultes [16, 17].

La surface musculaire et la surface corticale du radius sont étroitement corrélées chez tous les enfants, adolescents et adultes. La figure 4 montre ces corrélations chez les sujets de sexe masculin et féminin. Avant la puberté, la relation entre ces deux surfaces est identique chez les garçons et les filles, mais, après la puberté, la surface corticale est plus importante que la surface musculaire chez les filles comparativement aux garçons. Au stade pubertaire 3, la surface corticale est relativement plus grande 


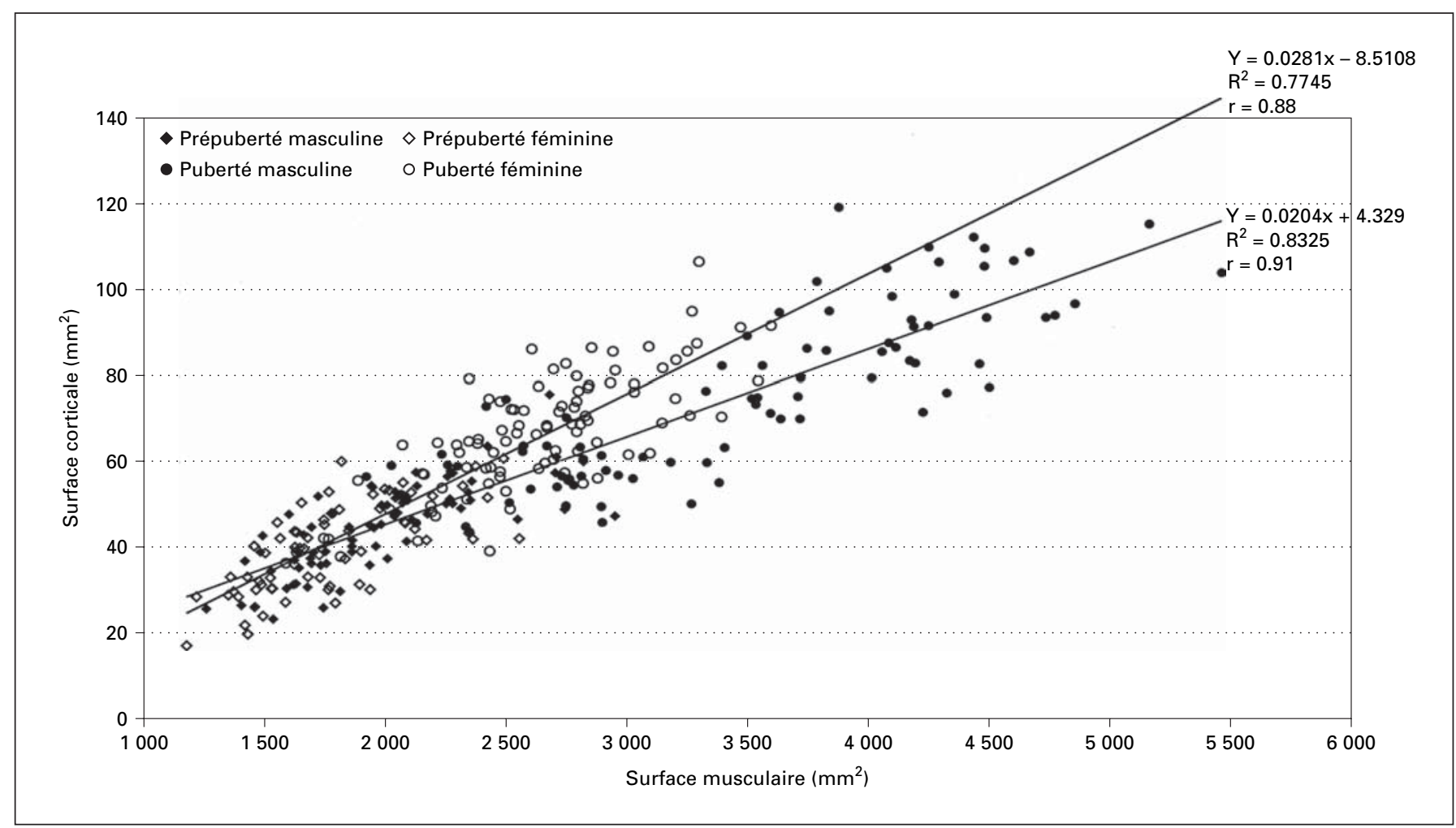

Fig. 4. Relation entre la surface osseuse corticale et la surface musculaire chez des garçons et des filles d'âge prépubertaire et pubertaire. Avec autorisation de Schoenau et coll. [16]; copyright 2000, The Endocrine Society.

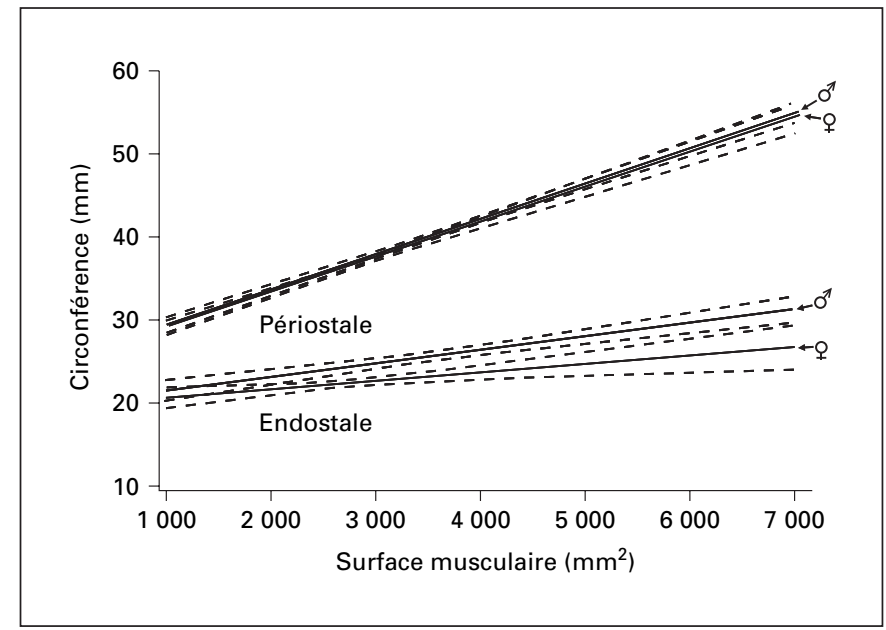

Fig. 5. Droites de régression et intervalles de confiance à $95 \%$ de la corrélation entre la surface musculaire et les surfaces périostales et endostales. Avec autorisation de Schoenau et coll. [16]; copyright 2000, The Endocrine Society.

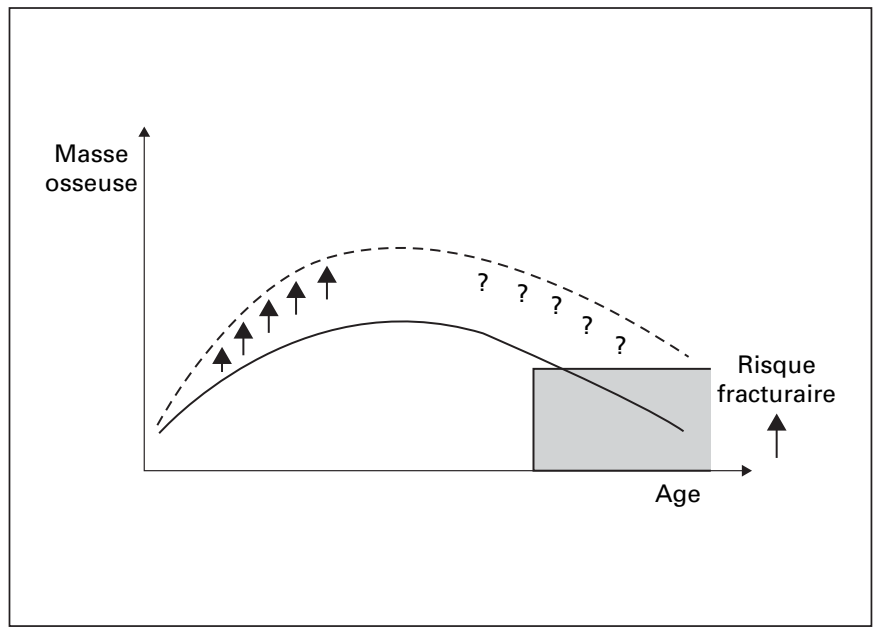

Fig. 6. Concept de pic de masse osseuse: prévention des fractures par amélioration de la masse osseuse individuelle au cours de l'enfance et de l'adolescence? D'après Springer Science and Business Media [Pediatr Nephrol 2004;19:825-831], avec autorisation. 


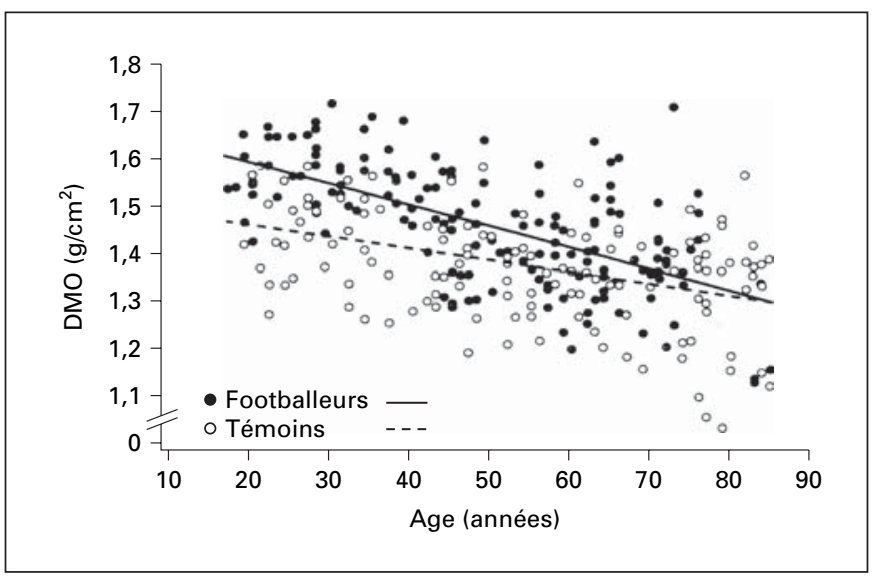

Fig. 7. Densité minérale osseuse (DMO) dans les membres inférieurs de footballeurs actifs et retraités comparativement à des témoins. D’après Johnston et coll. [19], avec autorisation.

chez les filles. La figure 5 indique la relation entre les circonférences périostales et endostales et la surface musculaire. La corrélation entre la surface musculaire et la circonférence périostale ne diffère pas significativement entre les sujets des deux sexes. En revanche, la relation entre la surface musculaire et la circonférence endostale diffère significativement en fonction du sexe. Ces données plaident en faveur des concepts proposés par Frost concernant le développement osseux au cours de l'enfance et de l'adolescence, et de la théorie de l'Utah portant sur la physiologie squelettique. La charge volontaire la plus importante s'exerçant sur les os provient des muscles. Afin d'adapter la résistance et la masse osseuses à ces derniers, des étendues particulières de seuils de contrainte déterminent les sites où le modelage ajoute de l'os et le renforce et les moments où le remodelage le conserve ou l'élimine, exactement comme les différents réglages d'un thermostat contrôlent le système de chauffage et de climatisation d'un domicile. Si les œstrogènes affectent la sensibilité du mécanostat en diminuant le seuil de remodelage à la puberté chez les filles, la masse osseuse devrait commencer à augmenter plus rapidement chez cellesci que chez les garçons ayant une force musculaire similaire, en raison d'une diminution de la déperdition osseuse dépendant du remodelage. Les résultats présentés ici complètent ceux des études de Zanchetta et coll. [14], Schiessl et coll. [15] et Ferretti et coll. [18] et confortent le concept cité.

\section{Masse osseuse maximale optimale au cours de l'enfance et de l'adolescence - Existe-t-il des effets à long terme?}

De nombreuses études ont analysé l'influence des apports en calcium sur le développement de la masse osseuse sur la base du concept généralement accepté de l'acquisition d'une valeur optimale de celle-ci (fig. 6). Une étude menée chez des enfants jumeaux a indiqué un effet positif mais modeste d'un accroissement des apports calciques sur la masse osseuse [19]. Il est intéressant de noter que les apports calciques n'ont exercé aucune influence sur la masse osseuse pendant la puberté, bien qu'ils soient largement considérés comme capitaux au cours de la période de croissance pubertaire. Un suivi supplémentaire de ces jumeaux a montré que les différences significatives de densité minérale osseuse constatées entre ceux recevant ou non une supplémentation orale en calcium disparaissaient après l'arrêt de celle-ci.

Une autre étude a permis d'observer que l'administration d'une supplémentation calcique contrôlée pendant 18 mois aboutissait à une masse minérale osseuse plus élevée, mais que ce gain disparaissait dans les 18 mois suivant l'arrêt de l'essai [20]. Des études de marqueurs biochimiques du modelage et du remodelage osseux ont montré qu'un apport en calcium réduisait le taux de remodelage mais ne modifiait pas le modelage [21].

Ces données sont compatibles avec l'hypothèse selon laquelle une supplémentation calcique diminuerait l'activité de remodelage. Une diminution des taux de remodelage pourrait survenir par l'intermédiaire d'une réduction de la concentration de l'hormone parathyroïdienne et aboutirait en une porosité corticale plus basse. Toutefois, ce gain apparent de masse osseuse disparaît totalement dès que le taux de remodelage augmente. Il est important de noter qu'une supplémentation calcique ne semble pas stimuler le modelage, qui est le principal processus d'augmentation de la résistance osseuse (épaisseur et surface corticales) au cours de l'enfance et de l'adolescence.

De même, les effets à long terme de l'activité physique pourraient être réversibles. Une étude menée chez des footballeurs a montré que l'activité physique au cours de la jeunesse conférait un bénéfice élevé et cliniquement pertinent en termes de densité minérale osseuse maximale, mais que l'arrêt de l'activité physique résultait en une diminution accélérée (fig. 7) [22]. Ainsi, les sujets qui avaient mis fin à leur activité sportive depuis plus de 35 ans et qui étaient âgés de 60 ans ou plus n’ont présenté aucun bénéfice résiduel significatif concernant la densité 
minérale osseuse. Le taux de fracture n'a pas été plus bas que celui attendu pour l'âge. Pajamaki et coll. [23] ont présenté des données similaires à la suite d'une expérimentation chez le rat. Les bénéfices osseux induits par l'activité physique diminuent si celle-ci est totalement arrêtée et, de ce fait, un entraînement permanent est probablement nécessaire au maintien jusqu'à l'âge adulte des effets positifs de l'activité physique exercée dans la jeunesse.

D'autre part, des études menées chez l'animal ont montré qu'une période de perturbation de l'acquisition de la masse osseuse au début de la vie pouvait ne pas résulter en une altération de la masse osseuse maximale [24]. L'étude a démontré que des surfaces du squelette juvénile ne sont pas totalement remodelées mais, en fait, remplacées en totalité tout au long de la croissance squelettique. Tandis que l'os juvénile grandit et que la cavité médullaire s'agrandit, l'os formé précocement dans la vie, quelle que soit sa qualité, est progressivement résorbé et remplacé par un os nouveau par l'intermédiaire du modelage. Ce mécanisme «répare» une insuffisance du développement osseux, après l'arrêt d'influences négatives.

En résumé, les effets à court terme sur la masse osseuse, qu'ils soient positifs ou négatifs, ne semblent pas exercer une influence quelconque à long terme. Lappareil squelettique semble s'adapter aux exigences actuelles plutôt qu'à celles d'une phase plus précoce du développement.

\section{Aperçu et nouveaux concepts}

Les suggestions et recommandations suivantes résument un nouveau concept qui repose sur les considérations ci-dessus, mais dont l'exactitude n'est pas démon- trée dans ses détails. La masse osseuse ne doit pas être reliée à l'âge, car des données de plus en plus nombreuses démontrent aujourd'hui qu'elle est plutôt liée à la dimension des os [25] ou à la fonction musculaire [25-31].

Selon cette analyse, il n'existe aucune «masse osseuse maximale». De nombreuses études sont en cours afin de déterminer si ces approches accroissent la sensibilité et la spécificité de la prévision de fractures chez un sujet.

De plus, le domaine de recherche de nombreux investigateurs en matière de physiologie osseuse se déplace de la masse osseuse vers la géométrie ou la résistance des os $[32,33]$. La masse osseuse est un marqueur indirect de la résistance osseuse, mais des techniques largement disponibles telles que l'absorptiométrie biphotonique aux rayons $\mathrm{X}$, la radiogramétrie et la tomodensitométrie peuvent également être utilisées afin de mesurer diverses variables de la géométrie osseuse telles que l'épaisseur et la surface corticales et le moment d'inertie. Les futures études permettront de savoir si l'analyse combinée de la géométrie et de la masse osseuses perfectionne ou invalide le concept de masse osseuse maximale.

A l'heure actuelle, l'analyse de la masse osseuse est très utile aux études épidémiologiques portant sur des facteurs pouvant influencer le développement osseux. La masse osseuse ne peut toutefois être considérée comme une mesure hautement sensible et spécifique du risque individuel de fracture. Le concept de masse osseuse maximale reposait sur la conception selon laquelle un développement optimal au cours de l'enfance et de l'adolescence préviendrait des fractures à l'âge adulte. Il est aujourd'hui clair que des os résistants chez un sujet jeune ne se traduisent pas automatiquement par une absence de fracture une fois celui-ci est âgé. Les os résistants pourraient toutefois le demeurer si le sujet maintient le mode de vie sain qui a initialement accru sa résistance osseuse. Il demeure à déterminer si cette hypothèse est correcte.

\section{Références}

1 Strock GA, Cottrell ER, Abang AE, et al: Childhood obesity: a simple equation with complex variables. J Long Term Eff Med Implants 2005;15:15-32.

2 Popkin BM: The nutrition transition: an overview of world patterns of change. Nutr Rev 2004;62:S140-S143.

3 Frost HM: Bone 'mass' and the 'mechanostat': a proposal. Anat Rec 1987;219:1-9.

4 Burr DB: Muscle strength, bone mass, and age-related bone loss. J Bone Miner Res 1997;12:1547-1551.

L’unité fonctionnelle ostéomusculaire
5 Schoenau E, Frost HM: The 'muscle-bone unit' in children and adolescents. Calcif Tissue Int 2002;70:405-407.

-6 Turner CH: Muscle-bone interactions, revisited. Bone 2000;27:339-340.

7 Frost HM: On our age-related bone loss: insights from a new paradigm. J Bone Miner Res 1997;12:1539-1546.

-8 Frost HM, Schonau E: The 'muscle-bone unit' in children and adolescents: a 2000 overview. J Pediatr Endocrinol Metab 2000; 13:571-590.
Cointry GR, Capozza RF, Negri AL, et al: Biomechanical background for a noninvasive assessment of bone strength and musclebone interactions. J Musculoskelet Neuronal Interact 2004;4:1-11.

10 Neu CM, Manz F, Rauch F, et al: Bone densities and bone size at the distal radius in healthy children and adolescents: a study using peripheral quantitative computed tomography. Bone 2001;28:227-232. 
-11 Neu CM, Rauch F, Manz F, Schoenau E: Modeling of cross-sectional bone size, mass and geometry at the proximal radius: a study of normal bone development using peripheral quantitative computed tomography. Osteoporos Int 2001;12:538-547.

12 Schoenau E, Neu CM, Rauch F, Manz F: The development of bone strength at the proximal radius during childhood and adolescence. J Clin Endocrinol Metab 2001;86: 613-618.

13 Schoenau E: From mechanostat theory to development of the 'Functional Muscle-BoneUnit'. J Musculoskelet Neuronal Interact 2005;5:232-238.

14 Zanchetta JR, Plotkin H, Alvarez Filgueira ML: Bone mass in children: normative values for the 2-20-year-old population. Bone 1995;16(suppl):393S-399S.

15 Schiessl H, Frost HM, Jee WS: Estrogen and bone-muscle strength and mass relationships. Bone 1998;22:1-6.

16 Schoenau E, Neu CM, Mokov E, et al: Influence of puberty on muscle area and cortical bone area of the forearm in boys and girls. J Clin Endocrinol Metab 2000;85:1095-1098.

$\checkmark 17$ Kersting M, Sichert-Hellert W, Lausen B, et al: Energy intake of 1 to 18 year old German children and adolescents. Z Ernahrungswiss 1998;37:47-55.
$>$

Ferretti JL, Capozza RF, Cointry GR, et al: Gender-related differences in the relationship between densitometric values of wholebody bone mineral content and lean body mass in humans between 2 and 87 years of age. Bone 1998;22:683-690.

19 Johnston CC Jr, Miller JZ, Slemenda CW, et al: Calcium supplementation and increases in bone mineral density in children. $\mathrm{N}$ Engl J Med 1992;327:82-87.

20 Lee WT, Leung SS, Leung DM, Cheng JC: A follow-up study on the effects of calciumsupplement withdrawal and puberty on bone acquisition of children. Am J Clin Nutr 1996; 64:71-77.

Slemenda CW, Peacock M, Hui S, et al: Reduced rates of skeletal remodeling are associated with increased bone mineral density during the development of peak skeletal mass. J Bone Miner Res 1997;12:676-682.

22 Karlsson MK, Linden C, Karlsson C, et al: Exercise during growth and bone mineral density and fractures in old age. Lancet 2000; 355:469-470.

23 Pajamaki I, Kannus P, Vuohelainen T, et al: The bone gain induced by exercise in puber ty is not preserved through a virtually lifelong deconditioning: a randomized controlled experimental study in male rats. J Bone Miner Res 2003;18:544-552.

-24 Gafni RI, McCarthy EF, Hatcher T, et al: Recovery from osteoporosis through skeletal growth: early bone mass acquisition has little effect on adult bone density. FASEB J 2002; 16:736-738.
25 Kroger H, Vainio P, Nieminen J, Kotaniemi A: Comparison of different models for interpreting bone mineral density measurements using DXA and MRI technology. Bone 1995; 17:157-159.

26 Molgaard C, Thomsen BL, Michaelsen KF: Influence of weight, age and puberty on bone size and bone mineral content in healthy children and adolescents. Acta Paediatr 1998;87:494-499.

27 Lu PW, Cowell CT, Lloyd-Jones SA, et al: Volumetric bone mineral density in normal subjects, aged 5-27 years. J Clin Endocrinol Metab 1996;81:1586-1590.

28 Schoenau E, Neu CM, Beck B, et al: Bone mineral content per muscle cross-sectional area as an index of the functional musclebone unit. J Bone Miner Res 2002;17:10951101.

-29 Rauch F, Schoenau E: The developing bone: slave or master of its cells and molecules? Pediatr Res 2001;50:309-314.

30 Finkelstein JS, Neer RM, Biller BM, et al: Osteopenia in men with a history of delayed puberty. N Engl J Med 1992;326:600-604.

31 Hogler W, Briody J, Woodhead HJ, et al: Importance of lean mass in the interpretation of total body densitometry in children and adolescents. J Pediatr 2003;143:81-88.

-32 Seeman E: From density to structure: growing up and growing old on the surfaces of bone. J Bone Miner Res 1997;12:509-521.

33 Seeman E: Periosteal bone formation - a neglected determinant of bone strength. N Engl J Med 2003;349:320-323. 